\title{
Transdifferentiation of bovine epithelial cells towards adipocytes in the presence of myoepithelium
}

\author{
Subi Sugathan ${ }^{1, a}$, Sung-Jin Lee ${ }^{1, a}$, Supriya Shiwani', and Naresh Kumar Singh ${ }^{2, *}$
}

* Corresponding Author: Naresh Kumar Singh Tel: +91-9554786939, Fax: +91-0542-2368993,

E-mail: naresh2101@gmail.com

${ }^{1}$ Department of Animal Biotechnology, College of Animal Life Sciences, Kangwon National University, Chuncheon 24341, Korea

2 Department of Veterinary Surgery and Radiology, Faculty of Veterinary and Animal Sciences, Institute of Agricultural Sciences, Banaras Hindu University, Varanasi-221005, Uttar Pradesh, India

a These two authors contribute equally to this work.

ORCID

Subi Sugathan

https://orcid.org/0000-0003-1174-9810

Sung-Jin Lee

https://orcid.org/0000-0001-9348-8356

Supriya Shiwani

https://orcid.org/0000-0002-6852-8384

Naresh Kumar Singh

https://orcid.org/0000-0001-6360-1610

Submitted Oct 25, 2018; Revised Feb 27, 2019; Accepted Mar 18, 2019
Objective: Orchastric changes in the mammary glands are vital, especially during lactation. The secretary epithelial cells together with the supporting myoepithelial and stromal cells function cordially to secrete milk. Increase in the number of luminal epithelial cells and a decrease in adipocytes are visible during lactation, whereas the reverse happens in the involution. However, an early involution occurs if the epithelial cells transdifferentiate towards adipocytes during the lactation period. We aimed to inhibit the adipocyte transdifferentiation of luminal cells by restraining the peroxisomal proliferator-activated receptor $\gamma(\operatorname{PPAR} \gamma)$ pathway.

Methods: Linolenic acid (LA) and thiazolidinediones (TZDs) induced adipogenesis in mammary epithelial cells were conducted in monolayer, mixed culture as well as in transwell plate co-culture with mammary myoepithelial cells.

Results: Co-culture with myoepithelial cells showed higher adipogenic gene expression in epithelial cells under LA+TZDs treatment. Increase in the expressions of PPAR $\gamma$, CCAAT/ enhancer-binding protein $\alpha$ and vimentin in both mRNA as well as protein levels were observed. Whereas, bisphenol A diglycidyl ether treatment blocked LA+TZDs induced adipogenesis, as it could not show a significant rise in adipose related markers. Although comparative results were found in both mixed culture and monolayer conditions, co-culture technic was found to work better than the others.

Conclusion: Antagonizing PPAR $\gamma$ pathway in the presence of myoepithelial cells can significantly reduce the adipogenisis in epithelial cells, suggesting therapeutic inhibition of PPAR $\gamma$ can be considered to counter early involution or excessive adipogenesis in mammary epithelium in animals.

Keywords: Transdifferentiation; Peroxisomal Proliferator-activated Receptor $\gamma$ (PPAR $\gamma$ ); Linolenic Acid; Luminal Epithelium; Adipogenesis; Myoepithelial

\section{INTRODUCTION}

The mammary fat pad adipocytes are essential for normal mammary gland development and vital for the postnatal development of mammary epithelium through the signal mediated morphogenesis of ducts [1-3]. Moreover, the function of adipocytes remain active in mammary gland throughout the lactation, however a visible adipocyte depletion is evident in lactation [4], which is further refilledduring involution [5]. On the other hand, the multiple layers cuboidal mammary epithelium will be composed to form the bulbous terminal end buds within the fat pad upon the pubertal hormonal responses [6]. Further proliferation and expansion of the epithelium and depletion of adipocytes will be happening due to reproductive hormonal stimulation during the lactation. However, when the nursing stops and suckling reduces, remodeling of mammary gland takes place through involution. In involution, some of the secretory epithelial will undergo apoptosis and the others will be 
redifferentiatedtowards adipocytes $[7,8]$.

As the mammary gland is derived from both endodermal and mesodermal origins [9], the epithelial proliferation and development are crucially depend on mesenchymal interactions [10]. The fat pad in mammary gland provides vital signals that trigger the ductal morphogenesis and mammary development and its absence in rodents prevented the normal mammogenesis [2]. Further, unlike the other fat tissues, mammary fat pad expresses certain hormone receptors [11], which are related to the mammary development and thus the thyroxine induced treatment intensified the hormonal adipoblastepithelial communication that is obligatory in each stage of developments in the gland [12].

Pubertal animals consist of adipose fat pad as a major compartment of mammary gland, whereas the mass of these fat pad tissues will be reduced by apoptosis and epithelial transdifferentiation in response to the hormonal regulations during pregnancy and lactation [13]. The lactating gland comprises of secretory epithelial tissue as dominant compartment, with a smaller fat pad. However, the fat pads are observed to be refilled through preadipocyte differentiation and adipogenesis of luminal cells during involution [4]. Therefore, to resist the luminal adipogenesis and thereby avoiding the precocious involution, we hypothesized to inhibit the major adipogenic specific peroxisomal proliferator-activated receptor $\gamma(\operatorname{PPAR} \gamma)$ pathway with the help of an antagonist namely bisphenol A diglycidyl ether (BADGE).

\section{MATERIALS AND METHODS}

\section{Chemicals, media, cells, and primers}

Dulbecco's modified Eagle's medium (DMEM, high glucose), penicillin and streptomycin (PS) were purchased from Welgene (Dalseogu, Daegu, Korea), whereas fetal bovine serum (FBS) and horse serum are respectively from hyclone laboratories Inc (Logan, UT, USA) from Gibco Technologies Inc (Grand Island, NY, USA). Retinoic acid, linolenic acid (LA), dexamethasone, insulin, biotin, ascorbic acid, pantothenic acid, ascorbic acid, paraformaldehyde, hematoxyline, eosin, Triton X-100, 4',6-diamidino-2-phenylindole dihydrochloride (DAPI) and fluoromount aqueous mounting media were obtained from Sigma Aldrich (St. Louis, MO, USA). Further, primary and secondary antibodies of both Donkey anti-goat immunoglobulin labelled with fluorescein isothiocyanate (IgG-FITC) and donkey anti-goat IgG-biotinylated were purchased from Santacruz biotechnology (Dallas, TX, USA) and primers from Macrogen.

\section{Mammary myoepithelial cell isolation}

The myoepithelial cells from bovine mammary gland were isolated enzymatically according to the standard protocol. Briefly, the dissected glandular tissues from mammary gland were washed with phosphate buffered saline (PBS) several times before removing the fat tissues. After the removal of fat as much as possible, the tissues were then minced into fine pieces and treated with collagenase type II $(300 \mathrm{U} / \mathrm{mL})$ and hyaluronidase $(100 \mathrm{U} / \mathrm{mL})$ at $37^{\circ} \mathrm{C}$ for $3 \mathrm{hrs}$. The digested cells were strained with $40 \mu \mathrm{m}$ cell strainer and washed with normal culture media and cultured in DMEM comprising 20\% FBS and 1.1\% PS. So isolated myoepithelial cells were proliferated and characterized using flow cyometric analysis with the help of BD FACSCalibur (BD-Biosciences, San Jose, CA, USA).

\section{Cell culture}

Co culture: Epithelial cells, which were stored in $\mathrm{LN}_{2}$, were seeded and proliferated (in DMEM containing 10\% FBS and $1.1 \%$ PS) till obtained a sufficient number of cells to perform the experiments. Total of $1 \times 10^{5}$ epithelial cells were seeded into the bottom layer, whereas the myoepithelial cells were seeded proportionally in the top mesh of the Co culture plate. When the epithelial cells reached near confluency, the differentiation treatments were started through the initial induction (DMEM containing 2\% horse serum, 1.1\% PS, $50 \mu \mathrm{M}$ ascorbic acid, $33 \mu \mathrm{M}$ biotin, $10 \mu \mathrm{M}$ acetic acid, $17 \mu \mathrm{M}$ pantothenic acid, $0.5 \mu \mathrm{M}$ 3-isobutyl-1-methylxanthine (IBMX), $1 \mu \mathrm{M}$ dexamethasone, $10 \mu \mathrm{M}$ insulin) for $24 \mathrm{hrs}$. Further, the treatment with $100 \mathrm{mM} \mathrm{LA}+10 \mu \mathrm{M}$ thiazolidinedione (TZD) and 100 $\mathrm{mM}$ LA+10 $\mu \mathrm{M}$ TZD+100 $\mu \mathrm{M}$ BADGE were carried out for 48 hours.

Mixed culture: equal number of both myoepithelial and epithelial cells were mixed together and seeded into the 100 $\mathrm{mm}$ plastic cell culture plates. On nearby confluency, the cells were treated with initial induction media for 24 hours, and followed by the treatments with $100 \mathrm{mM} \mathrm{LA}+10 \mu \mathrm{M}$ TZD and $100 \mathrm{mM} \mathrm{LA}+10 \mu \mathrm{M}$ TZD+100 $\mu \mathrm{M}$ BADGE for 8 days, with media change of every 48 hours.

Monolayer: Total of $1 \times 10^{6}$ epithelial cells were seeded into $100 \mathrm{~mm}$ dishes and the preconfluent dishes were treated with initial induction media followed by the $100 \mathrm{mM} \mathrm{LA}+10 \mu \mathrm{M}$ TZD and $100 \mathrm{mM} L A+10 \mu \mathrm{M}$ TZD+100 $\mu \mathrm{M}$ BADGE. The treatment carried out till day 6 with media change in every 48 hours.

\section{Flow cytometric analysis}

From the collected myoepithelial cells, small amount cells were fixed in $4 \%$ paraformaldehyde and permeabilized using triton X-100 (Sigma, Dorset, UK). These cells were stained with goat polyclonal primary antibodies, followed by the FITC conjugated donkey anti goat secondary antibodies. The flow cytometry was performed using FACS caliber and the data were analyzed using (Epics XL analyzer; Coulter Corporation, Miami, FL, USA). 


\section{Immunostaining}

The cell samples after the treatments were fixed in $4 \%$ paraformaldehyde before permeabilizing with Triton X-100. The permeabilized cells were incubated with skim milk blocker for blocking the unspecific proteins, followed by the staining with goat polyclonal primary antibodies (against mucin 1 [MUC1], keratin 18 [K18], K19, PPAR $\gamma$, CCAAT/enhancer-binding protein $\alpha[\mathrm{C} / \mathrm{EBP} \alpha]$ and vimentin proteins) and FITC labeled donkey anti goat secondary antibody. So stained cells were mounted in labeled glass slides with the help of flouromount gel. The prepared slides were visualized under the confocal laser microscope (Olympus FluoView FV1000, Tokyo, Japan) with the help of the Floueview confocal image analyzing software.

\section{Western blotting}

The total protein from the collected cell samples was isolated using the proprep protein isolation kit (iNtRON Biotechnology, Seongnam, Korea). The harvested samples were washed with ice cold PBS before lysing with Proprep buffer. The lysed cell suspension was centrifuged to collect the total protein containing supernatant. These protein samples were mixed with the sodium dodecyl sulfate (SDS) reducing gel loading dye and heated in $95^{\circ} \mathrm{C}$ for 5 mins and placed in ice till loading for the SDS-polycrylamide gel electrophoresis (SDS-PAGE). Further the separated proteins in the gel were transferred into nitrocellulose membrane under electro-blotting arrangement. The free plots in the membrane were then blocked with a blocker solution (5\% nonfat skim milk in PBS) to minimize the background. Further, the primary antibody incubation was carried out at $4^{\circ} \mathrm{C}$ overnight, followed by the biotinylated secondary antibody staining for 1 hour at room temperature. The biotin in the secondary antibody was then tagged with the streptavidin, which is conjugated with horse radish peroxidase enzyme. Finally, Opti-CN kit based detection was carried out and the visible bands were documented using an optical scanner. The protein bands were then analyzed using ImageJ software and the data were normalized to the internal loading control actin.

\section{Cytochemical staining}

Ayoub shklar: The staining sample cells were fixed in 4\% paraformaldehyde prior to staining. The fixed cells were treated with acid fuchsin stain for 5 mins, followed by the aniline blue and orange $\mathrm{G}$ (equal in volume) mixture for 40 mins. Further the stained cells were washed with $95 \%$ ethanol and air dried prior to take pictures.

Oil red O: The paraformaldehyde fixed cells were treated with 6:4 working solution of oil red O (ORO) for 10 mins. Further, washed thoroughly with water and taken the images under inverted light microscope. Finally, the stain was eluted out using 100\% isopropanol, for determining the ORO con- centration with the help of spectrophotometer.

Hematoxylin and eosin staining: Cells after fixing with paraformaldehyde were washed with distilled water, followed by the hematoxyline staining for 10 mins. The excess stains were washed out by washing with tap water and further stained with eosin for 5 mins. Finally, the stained samples were washed and dried using ethanol prior to take pictures under inverted light microscope.

\section{Reverse transcriptase polymerase chain reaction}

The total RNA from the harvested samples were extracted using trizol reagent (Invitrogen; Life Technologies Inc., Grand Island, NY, USA). cDNA synthesis from the collected total RNA performed using the reverse transcription kit (RiverTra Ace, Toyobo, Osaka, Japan), under the standard protocols of manufacturer. So synthesized cDNA samples were used for the polymerase chain reaction (PCR) amplification under the programmed conditions, using different primers (Table 1), in the thermocycler. The standard program used for PCR amplification is, initial denaturation at $95^{\circ} \mathrm{C}$ for $5 \mathrm{mins}$, followed by $35 \mathrm{cycles}$ of $95^{\circ} \mathrm{C}$ for $30 \mathrm{~s}, 58^{\circ} \mathrm{C}$ to $63^{\circ} \mathrm{C}$ for $30 \mathrm{~s}$ and $72^{\circ} \mathrm{C}$ for $30 \mathrm{~s}$, and a final elongation at $72^{\circ} \mathrm{C}$ for 8 mins before storing on $4^{\circ} \mathrm{C}$. The amplified products were then run in ethidium bromide stained $1 \%$ agarose gel and visualized under UV transilluminator documentation system. The bands obtained from the gels were analyzed using ImageJ software, further, the data were normalized to the expression of internal loading control glyceraldehyde 3-phosphate dehydrogenase.

\section{Statistical analysis}

The treatments in different experimental techniques at different replicates were analyzed by analysis of variance, using the statistical software package SAS (SAS Inst. Inc., Cary, NC, USA). The significant differences were determined ( $\mathrm{p}<0.05$, $\mathrm{p}<0.01, \mathrm{p}<0.001)$ by the Duncan's multiple range tests using SAS.

\section{RESULTS}

The isolated myoepithelial cells showed predominant expressions of basal cell markers comparatively to the other mammary cell specific proteins. Significant expression of smooth muscke actin, K14, vimentin were visible in the isolated cells, whereas other luminal and stromal cell markers showed a little expression (Figure 1).

\section{Effect of treatments on luminal cells under the influence of basal cells in coculture conditions}

The epithelial cells while cocultured with the mammary myoepithelial cells adopted luminal fate in normal control conditions, where expressions of K18, K19, and MUC1 along with the basal marker K14 were visible significantly. However, there 
Table 1. Primers used for reverse transcriptase polymerase chain reaction analysis

\begin{tabular}{|c|c|c|}
\hline mRNA & Forward & Reverse \\
\hline PPAR $\gamma$ & 5'-ACGGGAAAGACGACAGACAAA-3' & 5'-ACGGAGCGAAACTGACACC-3' \\
\hline Adipo Q & 5'-GATCCAGGTCTTGTTGGTCCTAA-3' & 5'-GAGCGGTATACATAGGCACTTTCTC-3' \\
\hline LPL & 5'-TACCCTGCCTGAAGTTTCCAC-3' & 5'-CCCAGTTTCAGCCAGACTTTC-3' \\
\hline C/EBPa & 5'-AGTCCGTGGACAAGAACAGC-3' & 5'-GGTCATTGTCACTGGTCAGC-3' \\
\hline K14 & 5'-TGATCAGCAGCGTGGAAGAG-3' & 5'-TGATCAGCAGCGTGGAAGAG-3' \\
\hline SMA & 5'-GATCACCATCGGGAATGAACGC-3' & 5'-CTTAGAAGCATTTGCGGTGGAC-3' \\
\hline K17 & 5'-ACTTCCGCACCAAGTTTGAG-3' & 5'-GCTTTCATCTCCTCCTCGTG-3' \\
\hline E-cadherin & 5'-CCAGGTGACCACACTTGATG-3' & 5'-ATACACATTGTCCCGGGTGT-3' \\
\hline Integrin b1 & 5'-TGTCGAGTGTGTGAGTGCAA-3' & 5'-AGACTCCAAGGCAGGTCTGA-3' \\
\hline Loricrin & 5'-CACTCATCCTTCCTGGTGCT-3' & 5'-GCCCCCGGAATACTTGATAC-3' \\
\hline K18 & 5'-GCGAGAAGGAGACCATGCAA-3' & 5'-AGAATTTGCAAAAATCTGAGCCCT-3' \\
\hline MUC1 & 5'-CGCAGAACTACGCCAGTTTCC-3' & 5'-AGAGCGGGTGGTCATGGA-3' \\
\hline EpCAM & 5'-CGGTCAGTGCCAGTGTACTT-3' & 5'-TTGAAGAGCCCCTTGTCGTC-3' \\
\hline K8 & 5'-ACCGGAACATCAACCGTCTC-3' & 5'-TCCCGTAGGACGAAGTCAGT-3' \\
\hline Vimentin & 5'-CAAGTCCAAGTTTGCTGACC-3' & 5'-TCATGTTCTGAATCTCATCCTG-3' \\
\hline GAPDH & 5'-GGCGTGAACCACGAGAAGTATAA-3' & 5'-CCCTCCACGATGCCAAAGT-3' \\
\hline
\end{tabular}

PPAR $\gamma$, peroxisomal proliferator-activated receptor $\gamma_{;}$LPL, lipoprotein lipase; C/EBP $\alpha$, CCAAT/enhancer-binding protein $\alpha ;$ K14, keratin 14; SMA, smotth muscle actin; MUC1, mucin1; EpCAM, epithelial cell adhesion molecule; GAPDH, glyceraldehyde 3-phosphate dehydrogenase.

were no signs of trigger in adipogenic markers such as $\operatorname{PPAR} \gamma$, $\mathrm{C} / \mathrm{EBPa}$, vimentin detected on immunostaining (Figure $2 \mathrm{~A}$ ), immunoblotting (Figure 2C), and RT-PCR (Figure 2D). Further, the ayoub shklar staining showed positively stained
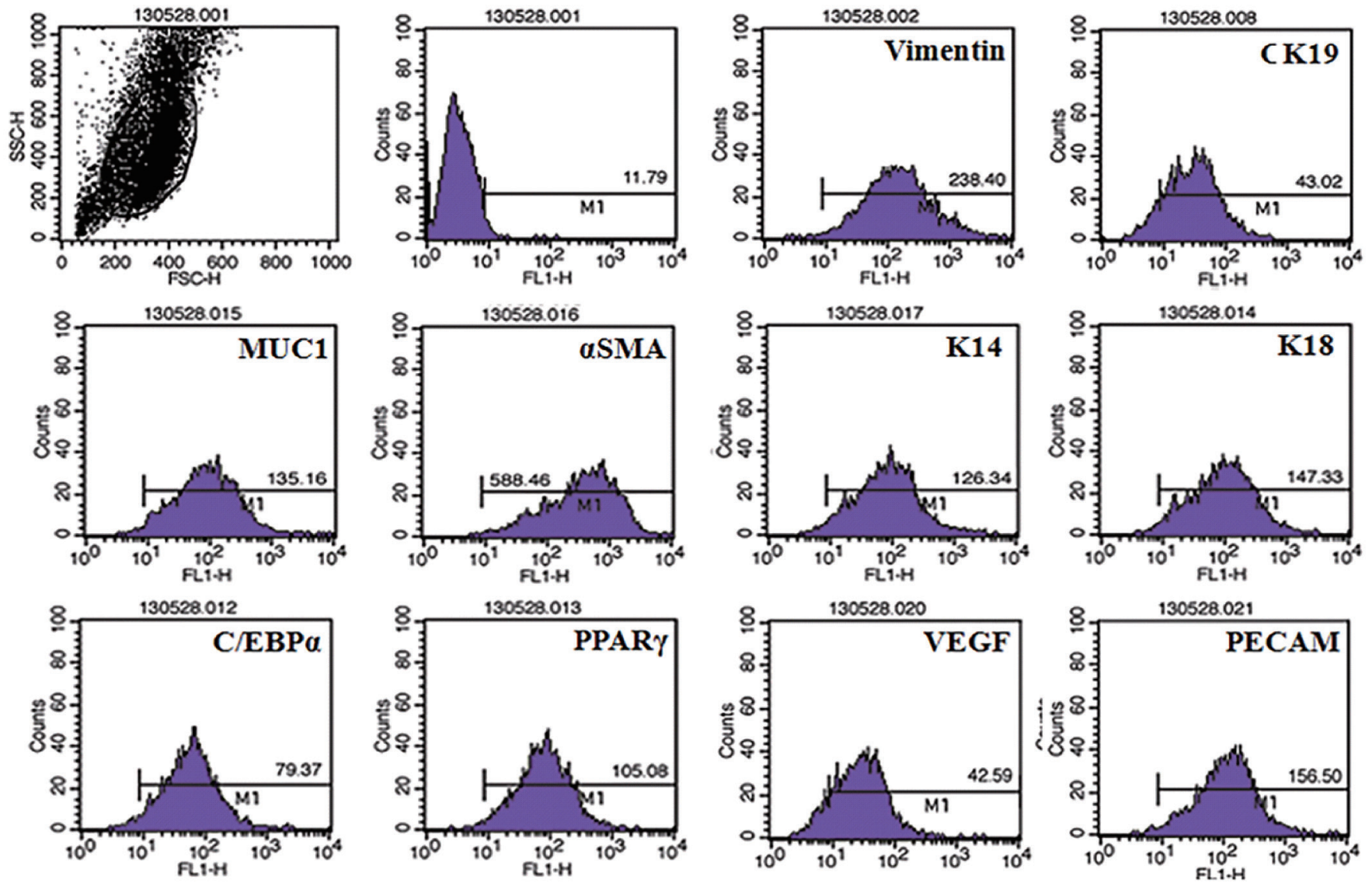

Figure 1. Flow cytometric characterization of mammary myoepithelial cells. Characterization of isolated bovine mammary myoepithelial cells using different markers. MUC1, mucin1; aSMA, a smotth muscle actin; K14, keratin 14; C/EBPa, CCAAT/enhancer-binding protein $\alpha$; PPAR $\gamma$, peroxisomal proliferator-activated receptor $\gamma$; VEGF, vascular endothelial growth factor; PECAM, platelet endothelial cell adhesion molecule. 
A

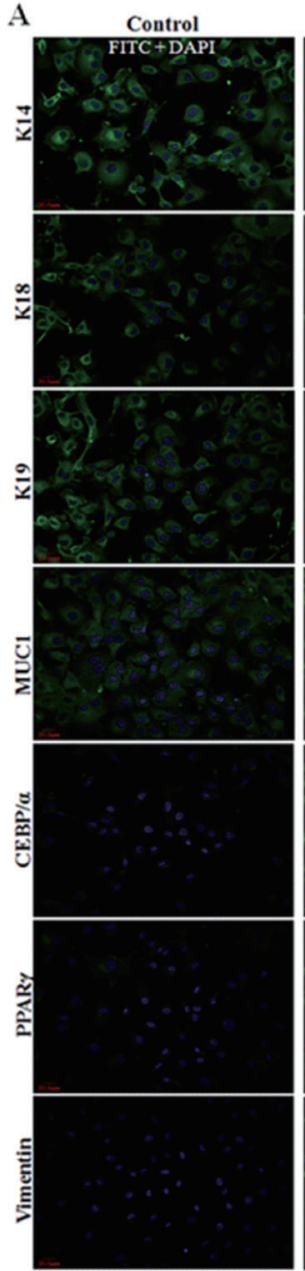

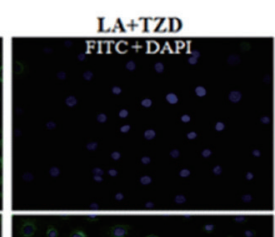
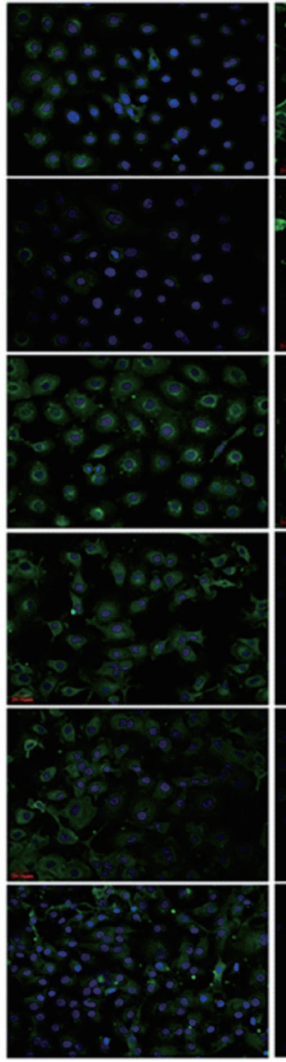

LA+TZD+BADGE
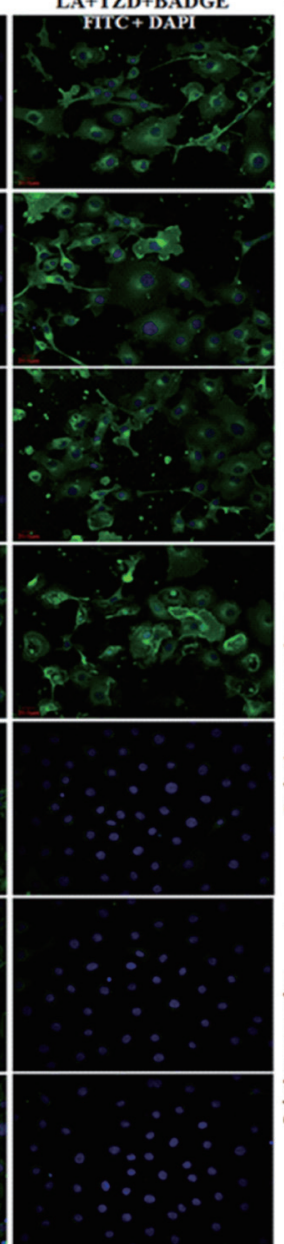

B

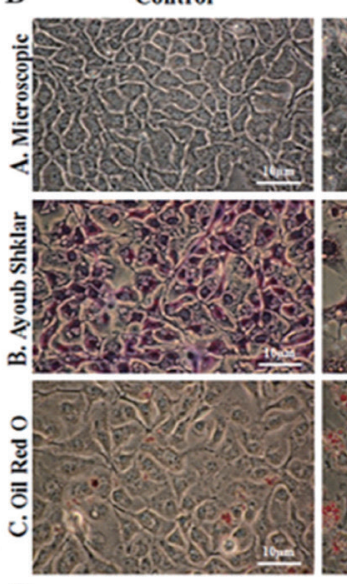

C $37=$ Control $\equiv \mathrm{EA}+\mathrm{TZD}=\mathrm{LA}+\mathrm{TZD}+\mathrm{BADGE}$
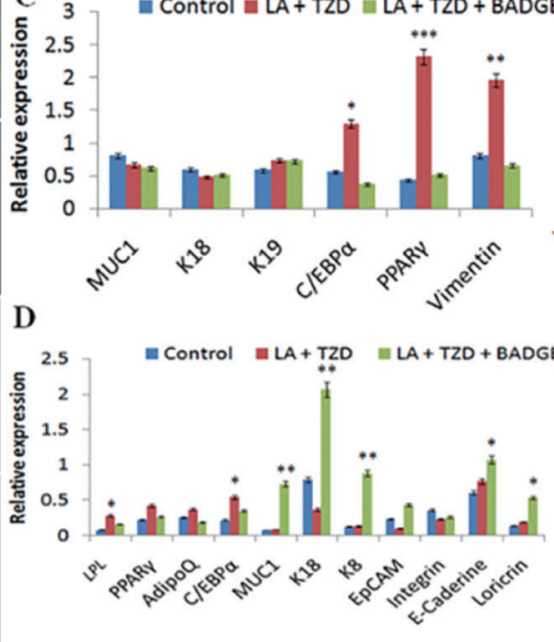

$\mathbf{L A}+\mathbf{T Z D}$
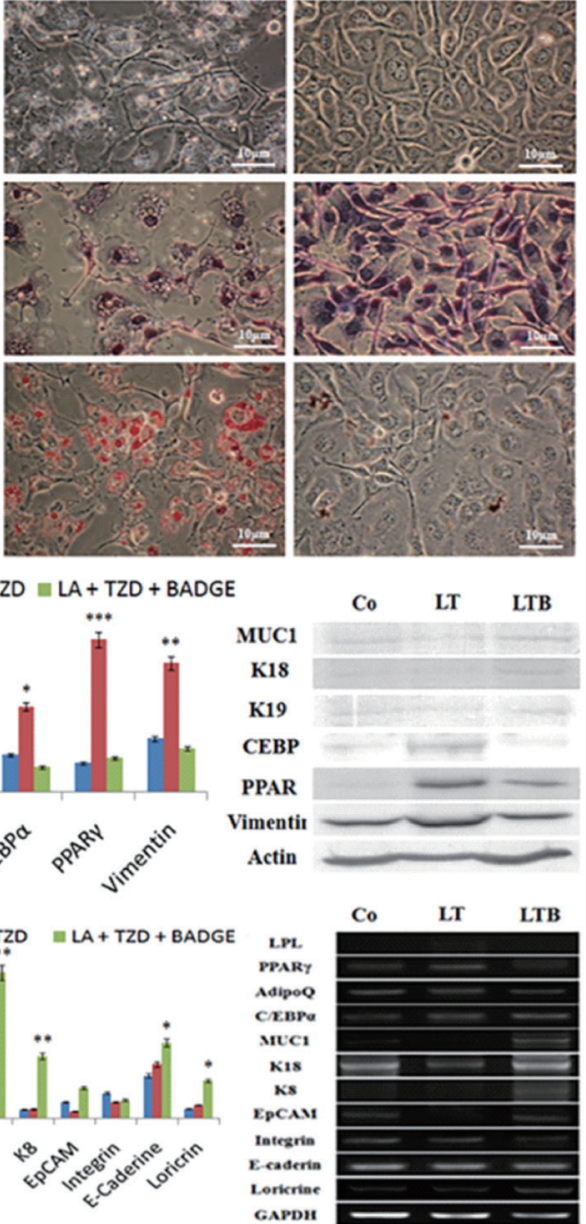

Figure 2. Adipocyte transdifferentiation of luminal cells under the influence of myoepithelial cells co culture. The adipogenic induction in luminal cells under the influence of myoepithelial cells revealed significant upregulation of fat specific markers. However, the inhibition of PPAR $\gamma$ signaling retarded adipose markers and enhanced/retained the luminal marker expression in immunostaining $(A)$, cytochemical staining $(B)$, immunoblotting $(C)$, and Reverse transcriptase polymerase chain reaction (D) analysis. $L A$, linolenic acid; TZD, thiazolidinediones; BADGE, bisphenol A diglycidyl ether; Co, control; LT, LA+TZD; LTB, LA+TZD+BADGE; PPAR $\gamma$, peroxisomal proliferator-activated

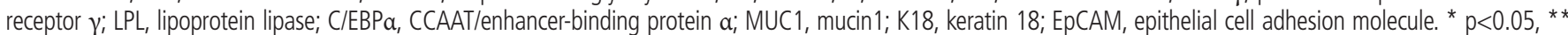
$p<0.01,{ }^{* \star *} p<0.001$.

epithelial cells and ORO unstained cells under control groups. In contrast, the LA+TZD treatment in luminal cells silenced the epithelial factors and enhanced the adipogenic transition. Under this treatment, intake of lipid droplets as well as the expressions of adipogenic markers was detected significantly enhanced.

However, the addition of BADGE in the induction media blocked the adipogenic impulse of luminal cells. The treatment of BADGE disrupted the PPAR $\gamma$ expression and, thereby, inhibited the other adipogenic factors such as $\mathrm{C} / \mathrm{EBP} \alpha$ and vimentin. Furthermore, the addition of BADGE in the adipogenic prompt media retained the luminal fate of epithelial cells, explained through the highly significant epithelial marker expressions in downstream analyses of genes and proteins as well as cytochemical stains (Figure 2B).
Effect of treatments on luminal and basal cells under mixed culture conditions

When the luminal and basal cells were cultured together in equal ratio, not much remarkable changes were noticed in control groups (Figure 3), where expressions of both luminal and basal markers showed balanced expressions (Figure 3A). However, when the cells were cultured under the presence of adipogenic inducers, disruption of luminal markers and insignificant change of basal K14 were noticed (Figure 3C). Further, the adipogenic specific markers like C/EBPa and PPAR $\gamma$ showed salient upregulation under the LA+TZD treatment (Figures 3A, 3C, 4) and the cytochemical staining of adipogenic induced cells observed tightly packed with the lipid droplets, though the cells likely myoepithelial in morphology rarely showed lipid inside. In addition, ayoub shklar and H\&E staining showed negatively stained for epithelium 

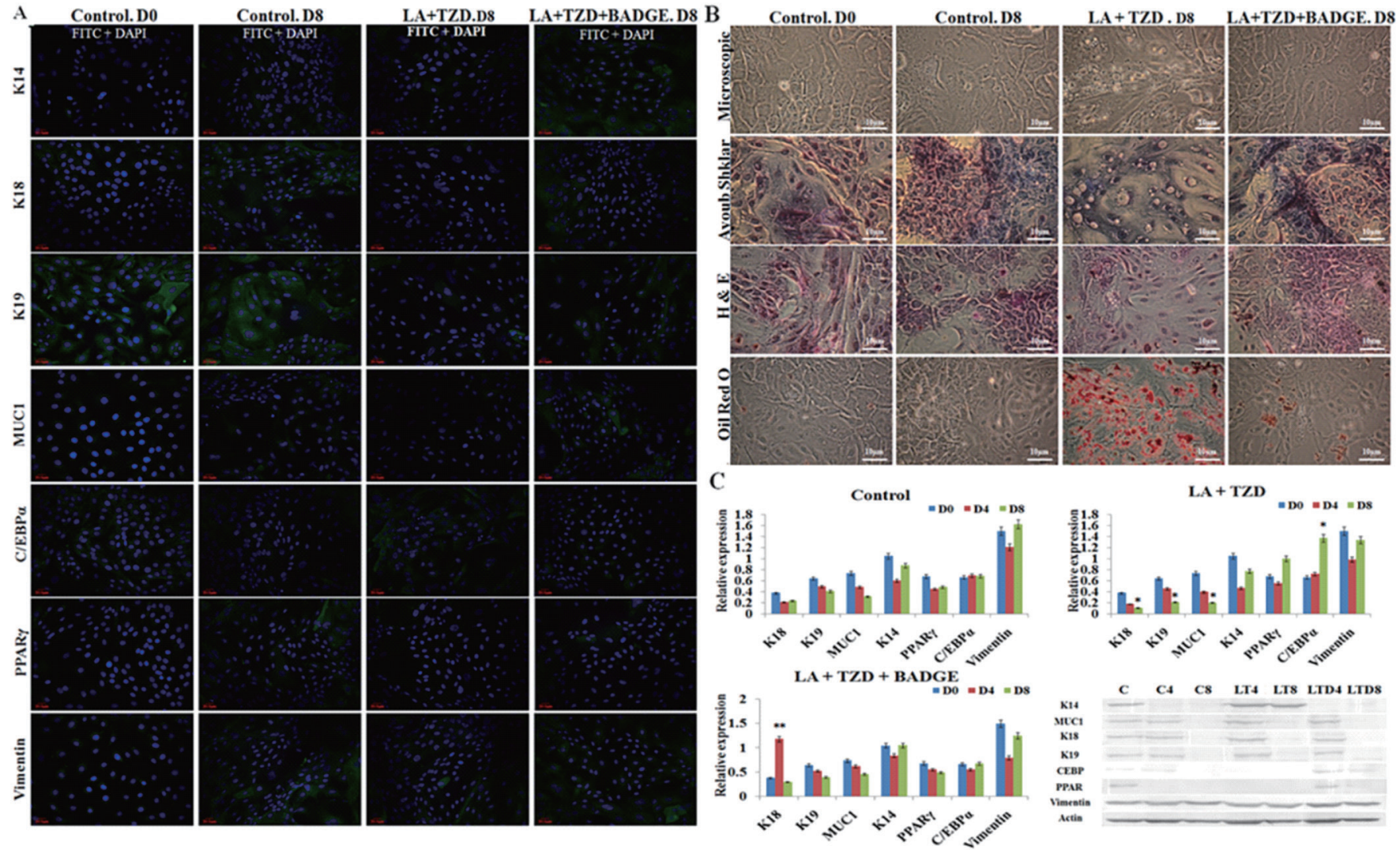

Figure 3. Adipogenic induction of luminal cells together with the myoepithelial cells in mixed culture. (A) and (C) The myoepithelial, epithelial and adipose specific marker protein expressions were analyzed through immunostaining and immunoblotting, where, downregulation of epithelial proteins in the control group (CO-C8) the, LA+TZD (LT4, LT8) and LA+TZD+BADGE (LTD4, LTD8) treated cells were monitored, thought the step up regulations in myoepithelial K14 in LTB and adipose proteins in LT treated cells were perceived. The documented bands within the membrane were analyzed through imageJ software and normalized to the internal loading control Actin ${ }^{*} \mathrm{p}<0.05$, ** $p<0.01)$. (B) Morphological alterations as well as cytological changes in mixed culture cells under adipogenic inductions were evaluated using microscopic observations of ayoub shklar, H\&E as well as oil red 0 stainings. Where smaller lipid intakes were shown in luminal cells under LA+TZD treatment, however, in the PPAR $\gamma$ blocked treatment, just like control, did not show any sign of adipose conversion even in the presence of LA+TZD. Further, ayoub shklar and H\&E stained images showed fading luminal factors under PPAR $\gamma$ active groups and higher luminal stains under PPAR $\gamma$ inhibition. LA, linolenic acid; TZD, thiazolidinediones; BADGE, bisphenol A diglycidyl ether; H\&E, hematoxylin and eosin; PPAR $\gamma$, Peroxisomal proliferator-activated receptor $\gamma ; \mathrm{K} 18$, keratin 18; MUC1, mucin1; C/EBPa, CCAAT/enhancer-binding protein a.

after treatment (Figure 3B).

On the other hand, the disruption of PPAR $\gamma$ signaling inhibited the adipogenic conversion in epithelial cells neighboring myoepithelium. The eight days of treatment could not trigger the adipogenic fate of luminal cells, whereas they maintained their own lineages under this particular treatment group. Changes in epithelial as well as basal markers were detected insignificant (Figures 3A, 3C, 4), moreover, cytochemical staining revealed the nondifferentiated cells in all ayoub shklar, $\mathrm{H} \& \mathrm{E}$ as well as ORO (Figure $3 \mathrm{~B}$ ).

\section{Effect of treatments on luminal epithelial cells in monolayer}

When the bovine mammary luminal cell cultures in monolayer, expressions of luminal markers, K18, K19, and MUC1 were monitored along with the slight expressions of adipocyte PPAR $\gamma$ and $\mathrm{C} / \mathrm{EBP} \alpha$ (Figure 5A). However, the treatment of $\mathrm{LA}+\mathrm{TZD}$ on luminal cells enhanced the adipogenic marker expressions significantly $(\mathrm{p}<0.05)$ (Figure $5 \mathrm{~A}, 5 \mathrm{C}, 6)$, thereby the lipid droplet formation within the cells were also intensified (confirmed by the elution index data for ORO staining). Moreover, the makeover of blue stains replacing the epithelial specific reddish dye explains the transdifferentiation of luminal cells from the epithelial lineage (Figure 5B). However, we did not attain the complete transition towards adipocyte lineage, where the expressions of luminal markers were also existed within the treated cells. Immuno-blotting data showed significant $(\mathrm{p}<0.05)$ step up regulation of PPAR $\gamma$ was observed in the treated cells, whereas the terminal adipogenic marker $\mathrm{C} / \mathrm{EBP}$ a expression was not significant. In contrast, the RNA analysis revealed significance in $\mathrm{C} / \mathrm{EBP} \alpha$ regulation and silencing of epithelial markers (Figure 6).

Conversely, the treatment with BADGE inhibited both luminal and adipose specific markers in epithelial cells. The PPAR $\gamma$ antagonist disrupted the adipogenic fate even in the presence of adipogenic inducers, moreover, fading off of K18, 

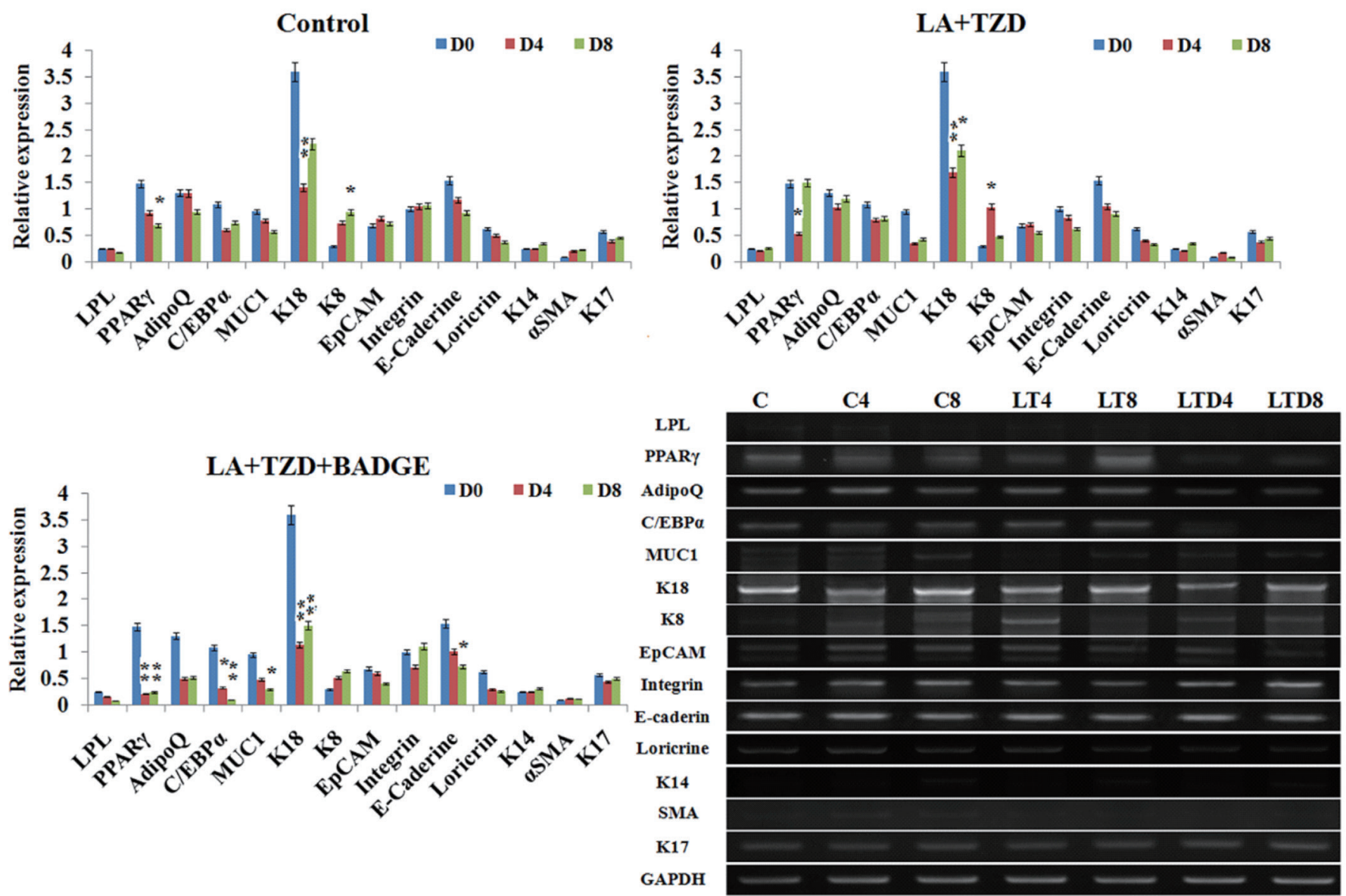

Figure 4. RT-PCR analysis for assessing variation in gene expressions upon adipocyte inductions in Epithelial and myoepithelial mixed culture. Adipocyte, epithelial and myoepithelial specific marker expressions under varied conditions of adipogenic inductions were detected using RT-PCR, where unchanged or negligible variations in epithelial marker gene expressions were observed in control (CO-C8) as well as PPAR $\gamma$ inhibited induction condition (LTB4, LTB8), in which, the adipocyte markers showed the gradual arrest of their expressions. However, in LA+TZD treatment (LT4, LT8), neither significant upregulation, nor downmodulations of adipocyte genes were observed. Although, slight downregulations of epithelial genes were detected. The normalization of each band was done with the expression of internal loading control GAPDH. * $p<0.05,{ }^{* *} p<0.01$. RT-PCR, reverse transcriptase polymerase chain reaction; PPAR $\gamma$, peroxisomal proliferator-activated receptor $\gamma ;$ LA, linolenic acid; TZD, thiazolidinediones; GAPDH, glyceraldehyde 3-phosphate dehydrogenase; LPL, lipoprotein lipase; C/EBPa, CCAAT/enhancer-binding protein $\alpha$; MUC1, mucin1; K18, keratin 18; K8, keratin 8; EpCAM, epithelial cell adhesion molecule; K14, keratin 14; aSMA, a smotth muscle actin; K17, keratin 17.

K19, and MUC1 under BADGE was noticeable (Figure 5A, 5C, 6). Nevertheless, vimentin expression showed positively regulated under this particular treatment. Furthermore, the ayoub shklar staining gave competable similarities with the control epithelial cells. In addition, ORO staining and its elution index revealed the non adipogenic cells without any stained lipid droplets (Figure 5B) under the BADGE treatment.

\section{DISCUSSION}

Plasticity of the mammary cell types plays an interesting part in mammary gland evolution, where the hormone regulated changes in luminal cells and stromal adipoblasts are remarkable during pregnancy, lactation and involution [13]. The major portion of mammary gland of a virgin animal covered by adipoblast, which will then make over to $90 \%$ of secretory epithelial cells during pregnancy and lactation by the transdifferentiation of adipoblast towards epithelium. However, the postlactational period refills the stromal fat pad through adipogenesis of luminal cells [13].

Our results provide the evidence that luminal epithelial cells under adipogenic inducers, such as LA, TZD's [14,15] can transdifferentiate to the adipocytes, however the PPAR $\gamma$ inhibition restricted the adipogenesis conversion in luminal epithelial cells. The TZD+LA treatment in luminal cells induced the PPAR $\gamma$ pathway that initiates the process of adipogenesis through lipid accumulation, which will be backed up by the terminal differentiator $\mathrm{C} / \mathrm{EBPa}[16,17]$. Moreover, these two genes together enhances the expressions of hundreds of other adipose specific genes those regulates lipid metabolism, lipid storage and adipokine secretion [18].

The changes in epithelial cells were detected by analyzing the difference in certain reliable luminal specific molecular 

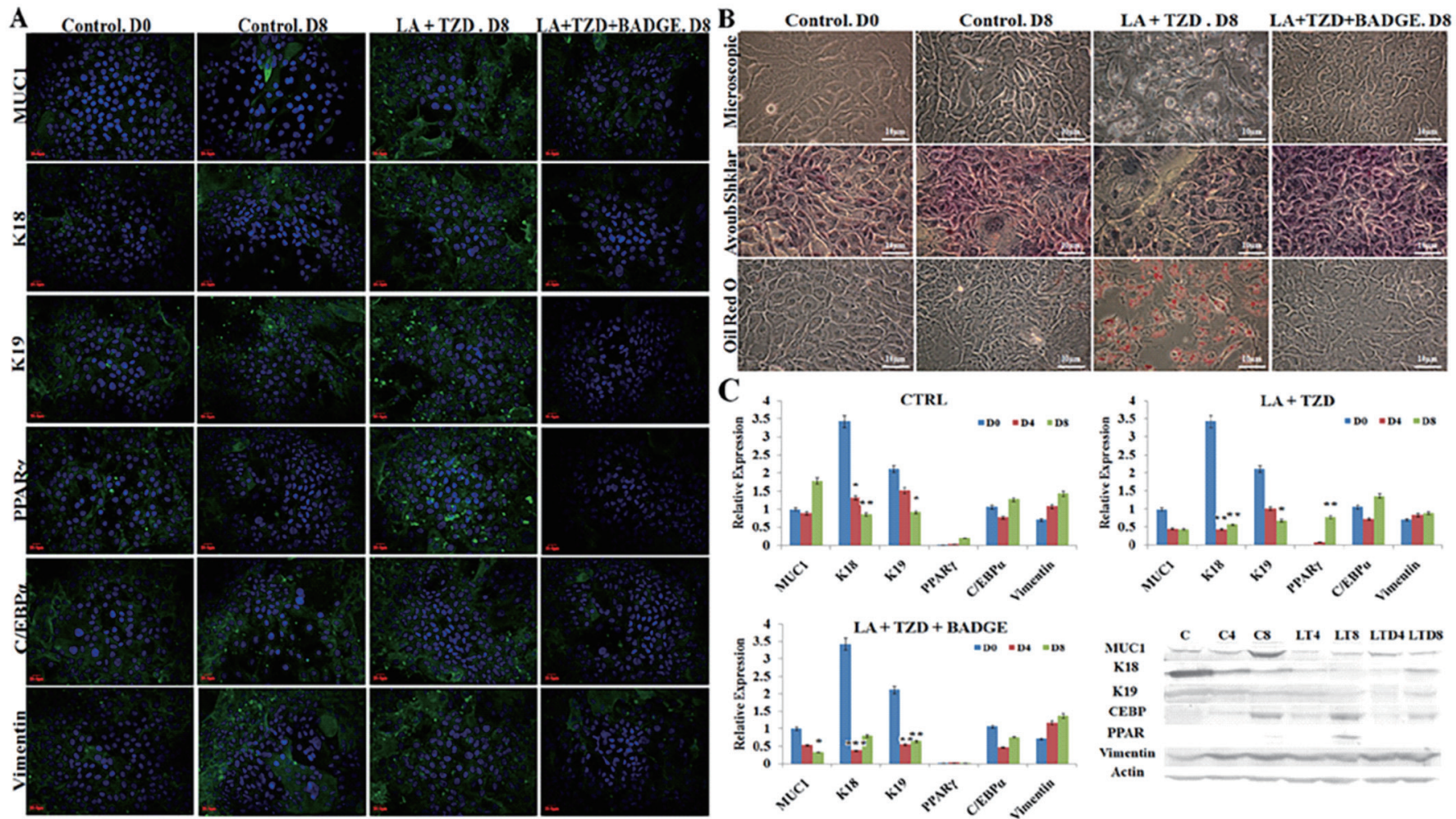

Figure 5. Adipogenesis of luminal cells in monlayer. (A) and (C) The epithelial and adipose specific marker protein expressions were analyzed through immunostaining and immunoblotting, where, adipogenic markers along with luminal markers were expressed under LA+TZD $(\mathrm{LT} 4, \mathrm{LT} 8)$ treatment, similarly the control cells (CO-C8) also expressed both kinds of markers but significantly lower adipogenic markers. However, the LA+TZD+BADGE (LTD4, LTD8) treatment restricted the adipogenic induction as epithelial markers were still evident. The documented bands within the membrane were analyzed through imageJ software and normalized to the internal loading contro Actin $\left({ }^{*} p<0.05,{ }^{* *} p<0.01\right)$. (B) The cells after the induction with adipogenic inducers monitored microscopically for morphological changes after ayoub shklar and oil red 0 staining. LA, linolenic acid; TZD, thiazolidinediones; BADGE, bisphenol A diglycidyl ether; MUC1, mucin1; K18, keratin 18; K19, keratin 19; PPAR $\gamma$, peroxisomal proliferator-activated receptor $\gamma_{;}$C/EBPa, CCAAT/enhancer-binding protein $\alpha$.

markers such as MUC1, K18, K19, and epithelial cell adhesion molecule (EpCAM). These molecular markers were abundantly expressed in luminal epithelial cells. EpCAM, which is localized in the basolateral membrane in normal epithelium, restricts their expression within epithelial cells. This molecular marker is being used to separate the completely differentiated epithelial cells from the cluster of cells [19]. Whereas, MUC1 is one of the mostly recognized molecular marker for epithelial cells, which is produced in the apical plasma membrane of lactating epithelial cells and involved in the cellular interactions as well as in signal transductions [20]. However, the $\mathrm{K} 18$ is one of the structural proteins which is being synthesized during the epithelial differentiation program, which is an epithelial specific molecular marker expressed in single layer epithelial tissues and characterize the differentiation compartment of mammary luminal epithelial cells [21]. The keratinocyte expression in the differentiating epithelia is initiated by the expression of primary cytokeratin proteins, for instance one of the simple keratins K18. Which is further supplemented by certain secondary keratinocytes, like [21] the luminal mammary epithelial cells are exclusively positive to the molecular markers such as EpCAM, MUC1, and K18, thus their consistant expressions are useful to identify the luminal epithelial cells in vivo [22], whereas their diminishing expressions under adipogenic induction denotes the change over of luminal fate.

We analyzed the changes in vimentin, which is a mesenchymal structural protein consisting of intermediate filament. The significant upregulation in vimentin under the adipogenic treatment shows the transition of luminal cells to mesenchymal adipocytes [23]. Similarly, the sustained E-caderin expression in control as well as BAGDE treated luminal cells explaines the unchanged luminal fate [23]. E-caderin is the transmembrane glycoprotein essential for the stable tissue architecture of epithelium. Whereas the expressions of E-caderin and vimentin are counter related, where one's expression suppresses the other's [24].

The conversion of preadipocyte to fully differentiated adipocytes is possible without the influence of any exogenous ligands. However, the adipogenesis of other cell types is entirely depend on the ectopic expressions of PPAR $\gamma$, which needs the exposure of certain exogenous ligands such as TZD's, 

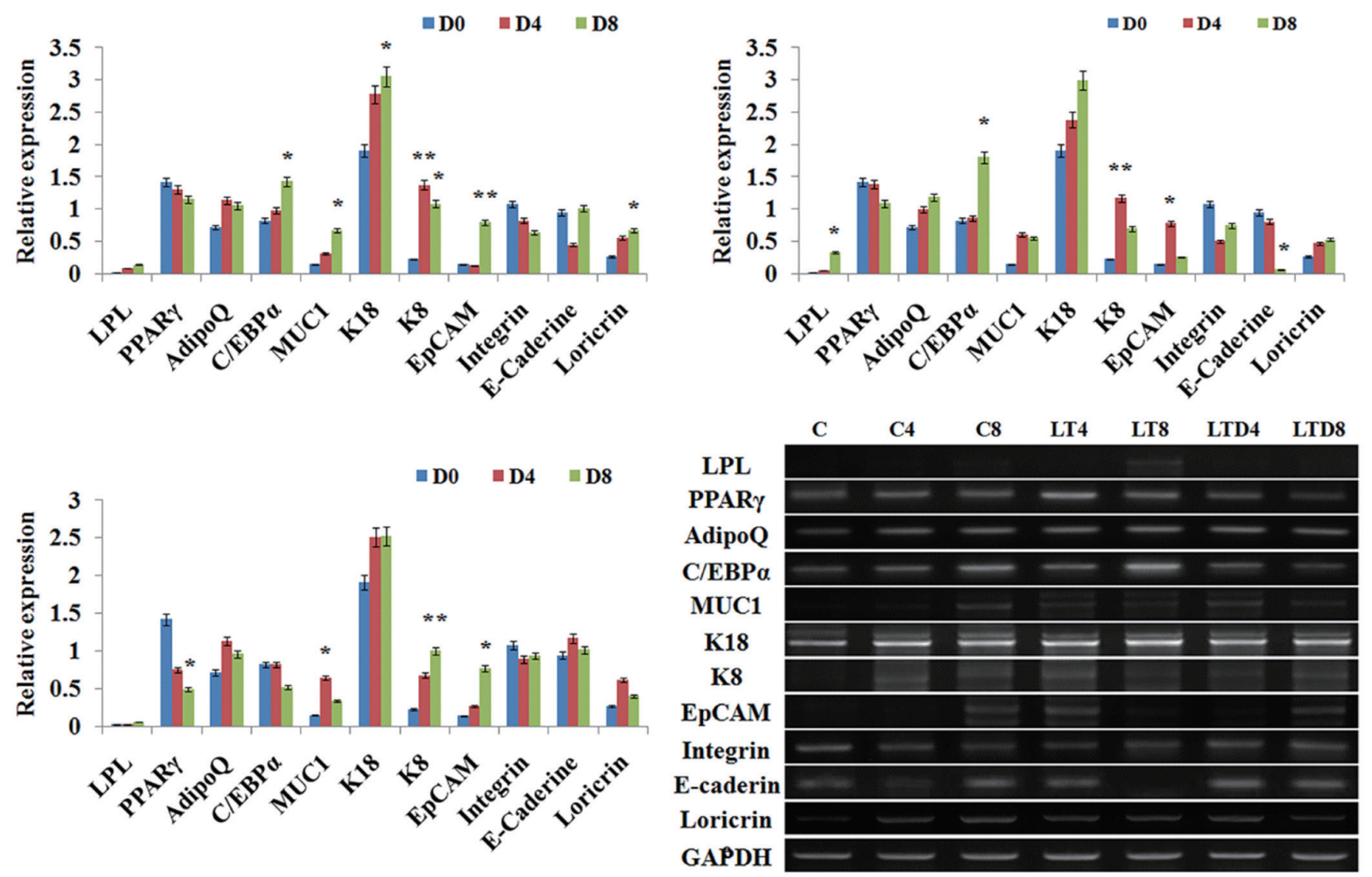

Figure 6. Reverse transcriptase polymerase chain reaction analysis of adipocyte and epithelial specific gene expressions in mammary epithelial cells under adipocyte differentiation conditions. Progressive upregulations of adipocyte genes in epithelial cells were observed under LA+TZD treatment (LT4, LT8), however, inhibition of PPAR $\gamma$ (LTD4, LTD8) blocked arrested the adipocyte gene expressions. Moreover, expressions of epithelial genes in control (C0-C8) cells were demodulated under adipocyte differentiation conditions. The normalization of each band was done with the expression of internal loading control glyceraldehyde 3-phosphate dehydrogenase $\left({ }^{*} p<0.05\right.$, $\left.{ }^{* *} \mathrm{p}<0.01\right)$. LA, linolenic acid; TZD, thiazolidinediones; LPL, lipoprotein lipase; PPAR $\gamma$, peroxisomal proliferator-activated receptor $\gamma ;$ C/EBPa, CCAAT/enhancer-binding protein $a$; MUC1, mucin1; K18, keratin 18; K8, keratin 8; EpCAM, epithelial cell adhesion molecule.

LA and so on [25]. Our initial induction media comprising of insulin, dimethyl sulfoxide and IBMX are the potent inducers if $\mathrm{C} / \mathrm{EBP} \beta$ and $\mathrm{C} / \mathrm{EBP} \delta$, whereas these factors eventually results in the ectopic expressions of two vital terminal adipogenic differentiation regulators PPAR $\gamma$ and $\mathrm{C} / \mathrm{EBPa}$ in non adipogenic cells [26]. Furthermore, the adipogenic differentiation at its molecular front is being operated in a feed-forward manner, where the initial induction of PPAR $\gamma$ influence the $\mathrm{C} / \mathrm{EBP} \alpha$ expression to produce the completely differentiated adipocytes [27].

The transition of epithelial cells towards adipocytes under the LA+TZD induction were further confirmed with the higher expressions of adipose specific lipoprotein lipase (LPL) as well as adipoQ. LPL is one of the early markers in adipogenic differentiation, which blocks the proliferation of cells and initiates differentiation [28]. This enzyme hydrolyzes the triglycerides and stores the free fatty acids within the tissue during the adipogenesis [29]. While, the secretary protein adipoQ, which is synthesized in adipocytes accelerates the adipogenic transition of luminal epithelial cells [30].
However, in mixed culture experiment, we did not observe any sign of adipogenesis in both luminal and basal cells except the initial lipid droplet formation. The mammary myoepithelial cells, which is also known as the natural tumor suppressor, block the mesenchymal transition of luminal cells [31]. Suggstively, the direct interaction of myoepithelial cells inhibited the luminal adipocyte conversion even under the potential adipogenic inducers. Although in monolayer experiments, the adipogenic induction resulted in lipid accumulation and significant upmodulations of PPAR $\gamma$, indicating initial adipogenesis [32], the complete conversion of luminal cells were not achieved within the experimental period. Nevertheless, the significant expression of $C / E B P \alpha$ gene on the final stages showed that the prolonged induction could provide completed transdifferentiation of luminal cells into adipocytes. Whereas, the presence of BAGDE in induction media restricted the initiation of adipogenesis and blocked the adipogenic transition altogether. Contrastingly, the upmodulation of vimentin was detected in the luminal cells, suggesting the possible transdifferentiation of mature luminal cells towards the vimentin 
postitve myoepithelium [33].

On the other hand, the treatment with a synthetic ligand (BADGE) of PPAR $\gamma$ antagonized the adipogenic differentiation in luminal cells, by competing with the agonists such as TZD's and LA. The complete blockage of PPAR $\gamma$ was not possible with the BADGE because of its low solubility and affinity compared to the agonists [34]. Furthermore, the expression of PPAR $\gamma$ at a minute level is essential for the successive lactation in ruminant as it helps to produce cystolic lipid droplets in luminal cells, which is essential for the milk fat formation and initiation of lactation [35]. Therefore, the use of synthetic antagonists like BADGE can block the excessive adipogenesis under lactation and a minimum of its expression to maintain efficient milk production.

In conclusion, the higher adipogenic transdifferentiation of luminal epithelium under adipogenic induction conditions can be inhibited through the treatment with synthetic PPAR $\gamma$ antagonistslike BADGE or its derivatives of similar chemical structure. Suggestively, the mammary gland/lactational defects such as invasion, precocious involution and so on can be regulated effectively by the inhibition of PPAR $\gamma$ pathway.

\section{CONFLICT OF INTEREST}

We certify that there is no conflict of interest with any financial organization regarding the material discussed in the manuscript.

\section{ACKNOWLEDGMENTS}

S Subi and SJ Lee has contributed equally to this paper as first author. The research was fully supported and facilitated from National Research Foundation of Korea (120120361).

\section{REFERENCES}

1. Hovey RC, Mcfadden TB, Akers RM. Regulation of mammary gland growth and morphogenesis by the mammary fat pad: a species comparison. J Mammary Gland Biol Neoplasia 1999; 4:53-68. https://doi.org/10.1023/A:1018704603426

2. Couldrey C, Moitra J, Vinson C, Anver M, Nagashima K, Green J. Adipose tissue: a vital in vivo role in mammary gland development but not differentiation. Dev Dyn 2002;223:459-68. https:// doi.org/10.1002/dvdy.10065

3. Fata JE, Werb Z, Bissell MJ. Regulation of mammary gland branching morphogenesis by the extracellular matrix and its remodeling enzymes. Breast Cancer Res 2004;6:1-11. https:// doi.org/10.1186/bcr634

4. Hovey RC, Aimo L. Diverse and active roles for adipocytes during mammary gland growth and function. J Mammary
Gland Biol Neoplasia 2010;15:279-90. https://doi.org/10.1007/ s10911-010-9187-8

5. Wang YY, Lehuédé $C$, Laurent $\mathrm{V}$, et al. Adipose tissue and breast epithelial cells: a dangerous dynamic duo in breast cancer. Cancer Lett 2012;324:142-51. https://doi.org/10.1016/j.canlet. 2012.05.019

6. Hennighausen L, Robinson GW. Singnaling pathways in mammary gland development. Dev Cell 2001;1:467-75. https:/doi. org/10.1016/S1534-5807(01)00064-8

7. Rucker EB 3rd, Hale AN, Durtschi DC, Sakamoto K, Wagner KU. Forced involution of the functionally differentiated mammary gland by overexpression of the pro-apoptotic protein bax. Genesis 2011;49:24-35. https://doi.org/10.1002/dvg.20691

8. Wiseman BS, Werb Z. Stromal effects on mammary gland development and breast cancer. Science 2002;296:1046-9. https://doi.org/10.1126/science.1067431

9. Watson CJ, Khaled WT. Mammary development in the embryo and adult: a journey of morphogenesis and commitment. Development 2008;135:995-1003. https://doi.org/10.1242/ dev.005439

10.Duss S, Brinkhaus H, Britschgi A, et al. Mesenchymal precursor cells maintain the differentiation and proliferation potentials of breast epithelial cells. Breast Cancer Res 2014;16:R60. https:// doi.org/10.1186/bcr3673

11. Cunha GR. Role of mesenchymal-epithelial interactions in normal and abnormal development of the mammary gland and prostate. Cancer 1994;74:1030-44.

12. Walden PD, Ruan W, Feldman M, Kleinberg DL. Evidence that the mammary fat pad mediates the action of growth hormone in mammary gland development. Endocrinology 1998; 139:659-62. https://doi.org/10.1210/endo.139.2.5718

13. Morroni M, Giordano A, Zingaretti MC, et al. Reversible transdifferentiation of secretory epithelial cells into adipocytes in the mammary gland. Proc Natl Acad Sci USA 2004;101:16801-6. https://doi.org/10.1073/pnas.0407647101

14. Ohyama M, Matsuda K, Torii S, et al. The interaction between vitamin A and thiazolidinedione on bovine adipocyte differentiation in primary culture. J Anim Sci 1998;76:61-5. https:// doi.org/10.2527/1998.76161x

15. Shin S, Ajuwon KM. Divergent response of murine and porcine adipocytes to stimulation of browning genes by 18-carbon polyunsaturated fatty acids and beta-receptor agonists. Lipids 2018;53:65-75. https://doi.org/10.1002/lipd.12010

16. Tamori Y, Masugi J, Nishino N, Kasuga M. Role of peroxisome proliferator-activated receptor- $\gamma$ in maintenance of the characteristics of mature 3T3-L1 adipocytes. Diabetes 2002;51:204555. https://doi.org/10.2337/diabetes.51.7.2045

17. Valet P, Tavernier G, Castan-Laurell I, Saulnier-Blache JS, Langin D. Understanding adipose tissue development from transgenic animal models. J Lipid Res 2002;43:835-60.

18. Farmer SR. Transcriptional control of adipocyte formation. Cell Metab 2006;4:263-73. https://doi.org/10.1016/j.cmet. 
2006.07.001

19. Kokubun K, Pankajakshan D, Kim MJ, Agrawal DK. Differentiation of porcine mesenchymal stem cells into epithelial cells as a potential therapeutic application to facilitate epithelial regeneration. J Tissue Eng Regen Med 2016;10:E73-83. https:// doi.org/10.1002/term.1758

20. Marin F, Luquet G, Marie B, Medakovic D. Molluscan shell proteins: primary structure, origin, and evolution. Curr Top Dev Biol 2007;80:209-76. https://doi.org/10.1016/S0070-2153 (07)80006-8

21. Buhler H, Schaller G. Transfection of Keratin 18 gene in human breast cancer cells causes induction of adhesion proteins and dramatic regression of malignancy in vitro and in vivo. Mol Cancer Res 2005;3:365-71. https://doi.org/10.1158/1541-7786. MCR-04-0117

22.Stingl J, Raouf A, Emerman JT, Eaves CJ. Epithelial progenitors in the normal human mammary gland. J Mammarry Gland Biol Neoplasia 2005;10:49-59. https://doi.org/10.1007/s10911005-2540-7

23. Kokkinos MI, Wafai R, Wong MK, Newgreen DF, Thompson EW, Waltham M. Vimentin and epithelial-mesenchymal transition in human breast cancer-observations in vitro and in vivo. Cells Tissues Organs 2007;185:191-203. https://doi.org/10.1159/ 000101320

24.Ackland ML, Newgreen DF, Fridman M, et al. Epidermal growth factor-induced epithelio-mesenchymal transition in human breast carcinoma cells. Lab Invest 2003;83:435-48.

25. Farmer SR. Regulation of PPAR $\gamma$ activity during adipogenesis. Int J Obes 2005;29(Suppl 1):S13-6.

26. Ahmed M1, Gaffen SL. IL-17 inhibits adipogenesis in part via $\mathrm{C} / \mathrm{EBP}$, PPAR $\gamma$ and Krüppel-like factors. Cytokine 2013;61: 898-905. https://doi.org/10.1016/j.cyto.2012.12.007

27. Rosen ED. The transcriptional basis of adipocyte development. Prostaglandins Leukot Essent Fatty Acids 2005;73:31-4. https:// doi.org/10.1016/j.plefa.2005.04.004

28. Salmerón C, Riera-Heredia N, Gutiérrez J, Navarro I, Capilla
E. Adipogenic gene expression in gilthead sea bream mesenchymal stem cells from different origin. Front Endocrinol (Lausanne) 2016;7:113. https://doi.org/10.3389/fendo.2016. 00113

29. Kolehmainen M, Vidal H, Ohisalo JJ, Pirinen E, Alhava E, Uusitupa MI. Hormone sensitive lipase expression and adipose tissue metabolism show gender difference in obese subjects after weight loss. Int J Obes Relat Metab Disord 2002;26:6-16. https://doi.org/10.1038/sj.ijo.0801858

30.Fu Y, Luo N, Klein RL, Garvey WT. Adiponectin promotes adipocyte differentiation, insulin sensitivity, and lipid accumulation. J Lipid Res 2005;46:1369-79. https://doi.org/10. 1194/jlr.M400373-JLR200

31. Polyak K, Hu M. Do myoepithelial cells hold the key for breast tumor progression? J Mammary Gland Biol Neoplasia 2005; 10:231-47. https://doi.org/10.1007/s10911-005-9584-6

32.Lee YJ, Choi HS, Seo MJ, Jeon HJ, Kim KJ, Lee BY. Kaempferol suppresses lipid accumulation by inhibiting early adipogenesis in 3T3-L1 cells and zebrafish. Food Funct 2015;6:2824-33. https://doi.org/10.1039/c5fo00481k

33. Péchoux C, Gudjonsson T, Ronnov-Jessen L, Bissell MJ, Petersen OW. Human mammary luminal epithelial cells contain progenitors to myoepithelial cells. Dev Biol 1999;206:88-99. https:// doi.org/10.1006/dbio.1998.9133

34. Wright HM, Clish CB, Mikami T, et al. A synthetic antagonist for the peroxisome proliferator-activated receptor gamma inhibits adipocyte differentiation. J Biol Chem 2000;275:18737. https://doi.org/10.1074/jbc.275.3.1873

35. McManaman JL, Palmer CA, Anderson S, Schwertfeger K, Neville MC. Regulation of milk lipid formation and secretion in the mouse mammary gland. In: Pickering LK, Morrow AL, Ruiz-Palacios GM, Schanler RJ, editors. Protecting infants through human milk. Advances in experimental medicine and biology. Boston, MA, USA: Springer; 2004. vol 554. pp. 263-79. https://doi.org/10.1007/978-1-4757-4242-8_22 